\title{
ANÁLISE DA PERDA DA FUNÇÃO RENAL EM JOVENS E ADULTOS USUÁRIOS DE ANABOLIZANTES SINTÉTICOS
}

\author{
ANALYSIS OF LOSS OF RENAL FUNCTION IN YOUNG AND ADULT USERS \\ OF SYNTHETIC ANABOLIZANTS
}

DOI: http://dx.doi.org/10.16891/2317-434X.v9.e2.a2021.pp1078-1085

Recebido em: 09.12 .2020 | Aceito em: 29.03.2021

\section{Ágata Bruna Neto Maia Pimentela*, Juliana Furlan Ravagnania, Robin Willian Soares Smith ${ }^{b}$, Aline Scharr Rodrigues ${ }^{c}$, Clarice Santana Milagres ${ }^{a, b}$}

\author{
Centro Universitário da Fundação Hermínio Ometto - FHOa \\ Faculdade São Leopoldo Mandic de Araras ${ }^{b}$ \\ Universidade de São Paulo, \\ Faculdade de Medicina de Ribeirão Preto, Departamento de Clínica Médicac \\ E-mail: agatabmaia@outlook.com
}

\section{RESUMO}

O uso de esteroides anabolizantes e suplementos alimentares apresentam crescente incidência, principalmente entre jovens atletas ou frequentadores de academias. A ingestão de alto teor de proteínas tem sido motivo de preocupação entre nefrologistas, já que tais ingestões potencializam as taxas de filtração glomerular e associam-se às hiperfiltrações glomerulares. Evidências recentes indicam que os esteroides anabolizantes são diretamente tóxicos para os glomérulos. Diante do exposto, o objetivo do presente estudo foi realizar uma busca por estudos científicos sobre a perda da função renal em jovens e adultos usuários de esteroides anabolizantes sem acompanhamento especializado. Foi realizada revisão dissertativa com artigos publicados entre o período de 2010 a 2020 nas bases de dados eletrônicos PubMed, Scielo e Science Direct. Os estudos encontrados em sua grande maioria foram trabalhos quantiqualitativos, além de revisões integrativas de literatura que apresentaram resultados originais e compilados de informações, respectivamente, sobre a temática analisada. Para melhor entendimento, a revisão foi dividida em duas abordagens: "anatomia renal e doença renal crônica" e "esteroides anabolizantes: uso indiscriminado, efeitos adversos e uso terapêutico". Estudos sobre a perda da função renal em jovens e adultos usuários de esteroides anabolizantes sem acompanhamento especializado são escassos, especialmente na literatura científica nacional. Contudo, os poucos dados encontrados reportam que o uso indiscriminado de esteroides anabolizantes pode causar lesões diversas ao organismo humano quando não acompanhados por um especialista. Quanto ao aparelho renal, este pode sofrer maiores danos, uma vez que é responsável pela filtragem e eliminação de impurezas do organismo

Palavras-chave: Anabolizantes; Adolescentes; Doença Renal Crônica.

\section{ABSTRACT}

The use of anabolic steroids and dietary supplements increases, especially among young athletes or gym-goers. The ingestion of high protein content has been the cause of concern among nephrologists, since such intakes increase as glomerular filtration rates and are associated with glomerular hyperfiltrations. Recent evidence indicates that anabolic steroids are directly toxic to glomeruli. Given the above, the objective of the present study was to conduct a search for scientific studies on the loss of renal function in young and adult adults on anabolic steroids without specialized monitoring. The dissertation was reviewed with articles published between 2010 and 2020 in the electronic databases PubMed, Scielo and Science Direct. Most of the studies found were quantitative-qualitative studies, in addition to integrative reviews of the literature, which presented original and compiled results of information, respectively, on the subject analyzed. For better understanding, a review was divided into two approaches: "kidney anatomy and chronic kidney disease" and "anabolic steroids: indiscriminate use, adverse effects and therapeutic use". Studies on the loss of renal function in young and adult users of anabolic steroids without specialized monitoring are scarce, especially in the national scientific literature. However, the few data found report that the indiscriminate use of anabolic steroids can cause injuries to the human body when not accompanied by a specialist. As for the renal system, it can suffer greater damage, since it is responsible for filtering and eliminating impurities from the body.

Keyword: Anabolics; Adolescents; Chronic Kidney Disease 


\section{INTRODUÇÃO}

A incidência e prevalência da Doença Renal Crônica em estágio cinco tem aumentado progressivamente a cada ano no Brasil e em todo o mundo. Segundo o estudo de Neves et al. (2020) sobre o censo brasileiro de diálise, a distribuição de pacientes em hemodiálise (HD) foi um pouco mais de $93 \%$, enquanto o restante encontrava-se em diálise peritoneal (DP). Estimase a incidência de novos pacientes com doença renal em diálise anual no país em torno de 42.500 , com tendências a aumentar. Calcula-se que mais de 130 mil pacientes estejam em tratamento dialítico e os gastos para manutenção dos programas de Terapia Renal Substitutiva (TRS), como hemodiálise, diálise peritoneal e transplante renal, consumam mais de dois bilhões de reais por ano, correspondente a mais de $10 \%$ de todo o orçamento do Ministério da Saúde. Do total desses serviços, $79 \%$ são financiados pelo Sistema Único de Saúde (SUS) e os outros 21\% resultam de convênios (NEVES et al., 2020; NEVES et al., 2021).

Em relação aos esteroides anabolizantes, estes se popularizaram primeiramente em academias de fisiculturismo, locais onde atletas desejam o corpo perfeito. Em programas de treinamentos exaustivos, "over training" e utilizando dietas restritas a proteínas, os efeitos obtidos sinérgicamente junto aos esteroides anabolizantes incluem ganhos em resistência, aumento de massa magra e estereótipo condizente com os padrões de beleza corporal masculinos. $\mathrm{O}$ uso de esteroides anabolizantes e suplementos alimentares apresentam crescente incidencia, principalmente entre jovens atletas ou frequentadores de academias, os quais têm como objetivo compensar deficiências dietéticas e melhorar o desempenho nas atividades realizadas (MEIRELLES; MENEZES; WEFFORT, 2011). Embora algumas pesquisas já apresentem os danos causados pela intensificação destes treinos, assim como mudanças nos hábitos de vida relacionados à continuidade deste padrão, o uso de esteroides anabolizantes tornou-se rotineiro entre estes individuos (ALMUKHTAR et al., 2015). Vale ressaltar que a grande parte destes usuários mantém uma dieta restrita e rígida para atletas de alto desempenho (PENDERGRAFT et al., 2014).

Os fisiculturistas frequentemente utilizam esteróides anabolizantes e suplementos dietéticos para adquirir força e volume corporal. Os principais suplementos não hormonais são proteínas, creatina e vitaminas. A ingestão de alto teor de proteína tem sido motivo de preocupação entre médicos nefrologistas, já que tais ingestões potencializam as taxas de filtração glomerular e associam-se às hiperfiltrações glomerulares (PARENTE FILHO et al., 2020). Evidências recentes indicam que os esteroides anabolizantes são diretamente tóxicos para os glomérulos (ALMUKHTAR et al., 2015).

Os esteróides anabolizantes sintéticos podem apresentar efeitos colaterais diversos, como infarto, esterilidade devido à atrofia de testículos, surtos psicóticos, gangrenas, insuficiência de órgãos, depressão, interrupção do crescimento em adolescentes, alteração do ciclo de menstruação, aumento de pêlos em mulheres e aumento de mamas em homens. Os principais efeitos adversos renais incluem elevações da creatinina sérica, da uréia e do ácido úrico, cujos valores retornam aos valores basais somente após a interrupção de tais drogas (PARENTE FILHO et al., 2020).

Segundo Ferreira et al. (2014), as recomendações terapêuticas dos Esteroides Anabolizantes podem ser aplicadas no hipogonodismo em adultos, algumas anemias, anabolismo proteico, angiodema hereditário, certas condições ginecológicas, déficit de crescimento e tratamento de osteoporose.

A nefropatia por ácidos biliares, também conhecida como nefrose colêmica ou nefropatia, ocorre também em usuários de anabolizantes sintéticos que possuem algum tipo de doenças hepáticas em estágios terminais e que são submetidos à terapia medicamentosa dessas drogas, não conseguindo excretar tais metabolitos, podendo, consequentemente, desenvolver a IRA e, em caso de lesão mais heterogênea, a síndrome hepatorenal e danos/necrose tubular aguda, combinada com diversas mudanças hemodinâmica com alguma contribuição direta de toxicidade relacionada a ácidos e diversas lesões em ductos biliares, tubulares e obstrução de néfrons, vindo assim a desenvolver a lesão renal (FERREIRA et al., 2014).

Em relação ao uso clínico, os esteroides anabolizantes possuem algumas funções importantes, como a reposição de testosterona nos casos em que haja um déficit relativo ao hormônio, determinada por motivo patológico, e também pode ser utilizado de forma medicinal, pois apresentam a propriedade de hipertrofiar os músculos. No entanto, este principal benefício também evolve a maior procura por atletas ou pessoas que almejam um melhor desempenho físico e resistência (FERREIRA et al., 2014; DAVANI-DAVARI; KARIMZADEH; KHALILI, 2019).

Diante do exposto, o objetivo do presente estudo foi realizar uma busca por estudos científicos sobre a perda da função renal em jovens e adultos usuários de 
esteroides anabolizantes sem acompanhamento especializado.

\section{METODOLOGIA}

Realizada revisão dissertativa com artigos publicados entre o período de 2010 a 2020. A opção por pesquisas dos últimos dez anos deve-se à busca por informações relevantes sobre o tema em questão e a construção de uma análise atual sobre a temática. Utilizou-se a bases de dados eletrônicos PubMed, Scielo e Science Direct, devido a facilidade de utilização dos filtros de seleção. Quanto à seleção dos descritores, foi utilizado o processo de revisão mediante consulta ao MeSH (Medical Subject Headings) e ao DeCS (Descritores em Ciências da Saúde). A utilização prédeterminada dos descritores em português, inglês e espanhol, abrangeram artigos que utilizaram "esteroides anabolizantes", "doença renal", “jovens adultos" e "adultos" em combinação. Também foram verificadas as referências dos artigos relevantes para identificar estudos potencialmente elegíveis, assim como foram utilizadas legislações, resoluções e normas técnicas para maior fidelização da revisão proposta.

A pesquisa realizada no PubMed adotou os critérios: título/abstract; publicado entre 2010 e 2020; texto completo; texto livre completo; humanos; escrito em inglês, português e espanhol; publicado em periódicos, jornais de artigos e revistas clínicas. Realizadas as seguintes combinações de descritores: anabolizantes, jovens, adultos e a expressão doença renal crônica. Pela pesquisa eletrônica foram revisadas para identificação de estudos adicionais.

\section{RESULTADOS E DISCUSSÃO}

Os estudos encontrados em sua grande maioria foram trabalhos quantiqualitativos, além de revisões integrativas de literatura que apresentaram resultados originais e compilados de informações, respectivamente, sobre a temática analisada. Para melhor entendimento, a revisão foi dividida em duas abordagens: "anatomia renal e doença renal crônica" e "esteroides anabolizantes: uso indiscriminado, efeitos adversos e uso terapêtico".

\section{Anatomia renal e Doença Renal Crônica}

Segundo Riella (2018), os rins são um par de órgãos situados na parede posterior do abdômen atrás do peritônio, em cada lado da coluna vertebral. No adulto seu peso varia de 115 a 170 gramas, com altura de aproximadamente $11 \mathrm{~cm}$, largura de $6 \mathrm{~cm}$, e espessura de $3 \mathrm{~cm}$. Os rins possuem três regiões principais: o córtex, que é a região mais externa; a medula, região central que se divide em externa e interna e, por fim, a papila, que é a ponta mais interna da medula inteira e drena para as bolsas.

\section{Hall (2017, p. 258) ainda descreve que:}

\begin{abstract}
Os dois rins se situam na parede posterior do abdome, fora da cavidade peritoneal. Cada rim de ser humano adulto pesa cerca de 150 gramas e tem o tamanho aproximado de uma mão fechada. O lado medial de cada rim apresenta região indentada chamada hilo. Pelo hilo passam a artéria e veia renais, vasos linfáticos, suprimento nervoso e o ureter, que carreia urina do rim para a bexiga. Na bexiga, a urina é armazenada e periodicamente eliminada do corpo. $\mathrm{O}$ rim é revestido por cápsula fibrosa resistente que protege as estruturas internas que são mais delicadas (HALL, 2017, p. 258).
\end{abstract}

Os rins possuem como principais funções a regulação da osmolaridade e dos volumes corporais; regulação do equilíbrio eletrolítico acido-básico; excreção de produtos metabólicos e substâncias estranhas; produção e secreção de hormônios; ajustagem do volume constante; regulação da pressão arterial sistêmica; regulação da homeostase hidroeletrolítica, incluindo o cálcio, o fósforo, o magnésio; e degradação de peptídeos circulantes, como a insulina. Essas funções são realizadas de forma integrada por diversas unidades funcionais denominadas nefrons que, formadas por outra unidade funcional chamada glomérulo, faz com que o fluxo sanguíneo que inicialmente passa por este, filtre o plasma e remova ou adicione água e solutos que posteriormente viram excreta, a urina, que é o produto final do trabalho conjunto dessas unidades (RIELLA, 2018).

A Doença Renal Crônica (DRC) consiste em uma lesão renal e perda progressiva e irreversível das funções renais glomerular, tubular e endócrina. O tratamento da DRC consiste em um procedimento através do qual uma máquina limpa e filtra o sangue, ou seja, faz a parte do trabalho que o rim doente não pode realizar (Riella, 2018). A evolução da função renal normal para lesão renal crônica sintomática ocorre através de uma série de fases, contabilizando cinco no total, que são definidas através de medidas de creatinina sérica a partir das quais as estimativas de redução na taxa de filtração glomerular são derivadas (KUMAR; ABBAS; ASTER, 2013).

Para Bastos; Bregman; Kirsztajn (2010), os rins são órgãos fundamentais para a manutenção da homeostase do corpo humano. Assim, não é surpresa que 
a diminuição progressiva da função renal implique em comprometimento de essencialmente todos os outros órgãos. Portanto, a função renal é avaliada pela filtração glomerular (FG) se constatada pela diminuição na doença renal, sendo correlacionada à perda das funções regulatórias, havendo uma queda nos índices de filtração de $15 \mathrm{ml} / \mathrm{min} / 1,73 \mathrm{~m} 2$, estabelecendo por sua vez falência renal.

As principais manifestações sistêmicas da DRC, segundo Kumar; Abbas; Aster (2013), estão relacionados à desequilibrios de líquidos e eletrólitos (desidratação, edema, hipercalemia, acidose metabólica); fosfato de cálcio e osso (hiperfosfatemia, hipocalcemia, hiperparatireoidismo secundário, osteodistrofia renal); hematológicos (anemia e diátese hemorrágica); cardiopulmonares (hipertensão arterial, insuficiência cardíaca congestiva, cardiomiopatia, edema pulmonar, pericardite urêmica); gastrointestinais (náuseas e vômitos, sangramento, esofagite, gastrite, colite); neuromusculares (miopatia, neuropatia periférica, encefalopatia) e dermatológicos (palidez, prurido, dermatite).

\section{Esteroides anabolizantes: uso indiscriminado, efeitos adversos e uso terapêutico}

Segundo Felício (2010), EAA (Esteroides Anabólicos Androgênico) são substâncias construtoras de tecido muscular cujos efeitos são similares aos da testosterona, e quando utilizados de forma abusiva, contribuem como um risco para a vida do indivíduo.

Desde o século XIX os esteroides anabolizantes já eram utilizados no tratamento de pacientes terminais, em especial com debilidades crônicas, queimaduras extensas, depressão e recuperação de procedimentos cirúrgicos críticos (FERREIRA et al., 2014). Contudo, a partir da década de 50 os esteroides anabolizantes passaram a ser prescritos devidamente pela medicina. Atualmente, são utilizados na assistência de deficiências androgênicas, como hipogonodismo, puberdade e retardo no crescimento, micropênis neonatal, deficiência hormonal parcial em homens idosos, deficiências secundárias a doenças crônicas, e contracepção masculina hormonal. Os esteroides anabolizantes também são usados no tratamento de osteoporose, em casos de anemias causadas por falha na medula óssea ou rins, câncer de mama, em pessoa do sexo masculino com estatura elevada e em muitos casos de obesidade (FERREIRA et al., 2014). Existem ainda relatos de uso de reposição de esteróides anabólicos via transdérmica em baixas doses para problemas cardiovasculares, possuindo efeitos antiterogênicos e como agentes antianginosos. Há estudos que relatam a utilização dos EAA (Esteroides Anabólicos Androgênicos) no tratamento de sarcopenia relacionadas ao vírus da imunodeficiência adquirida (HIV), fadiga em pacientes com diagnóstico de doença renal crônica submetido à diálise, sarcopenia relacionada à cirrose alcoólica e doença obstrutiva pulmonar crónica (PARENTE FILHO et al., 2020).

Atualmente, existe no mundo uma preocupação sociogovernamental sobre o uso abusivo dos EAA. Estudos mostram que a taxa de prevalência de EAA entre adolescentes variam de $2 \%$ a $12 \%$ para o sexo masculino e $0,5 \%$ a $2,5 \%$ para o sexo feminino. A diferença de prevalência entre os sexos está associada ao fato de que os usuários de EAA estão em busca de aumento de massa muscular, um efeito que, em sua maioria, é indesejável pelas mulheres. Dados mostram que nos EUA estima-se que 3,5 milhões de usuários já fizeram o uso dessa classe de droga em algum período da vida (DAVANI-DAVARI; KARIMZADEH; KHALILI, 2019). No Brasil, o levantamento sobre o uso de drogas psicotrópicas pelos jovens brasileiros em idade escolar, nas principais capitais nacionais, mostra que $2 \%$ deles já fizeram uso de esteroides anabolizantes (GRACELI et al., 2010).

A incidência do uso de EAA nos últimos anos tem crescido alarmantemente, constatando uma frequência entre os usuários de 20 anos de idade, $5 \%$ de 20 a 24 anos e aumentando de $18 \%$ para $46 \%$ nos usuários de 25 a 29 anos. Após essa idade, o uso passa a ser de $13 \%$ para os que possuem faixa etária de 35 a 39 anos, declinado posteriormente a $5 \%$ para os que possuem mais de 40 anos, sendo a maioria do sexo masculino (VENÂNCIO et al., 2010; SANTOS, 2012).

Após serem processadas, as fórmulas dos EAA dão origem a produtos como: Oxandralona (Anavar®), Estanazolol (Winstrol®), Nandrolona (Deca Durabolin $®)$, Metandionona (Dianabol®), Metanolona (Primobolan () ), Ciprionato de testosterona (Deposteron $\AA$ ), Decanoato (Deca-Durabolin $®$ ), Oximetalona (Hemogenin $®)$, testosterona e outras substâncias destinadas ao uso veterinário, principalmente equino. $\mathrm{O}$ uso destes produtos, utilizados por via oral e parenteral de forma errônea por usuários que desconhecem os efeitos psíquicos e físicos destes compostos é visto nos dias atuais (FERREIRA et al., 2014).

Segundo relatos da literatura, os jovens e adultos jovens utilizam EAA de três maneiras: 1). "Ciclo", referindo a qualquer período de utilização de tempos em tempos, que varia de quatro a 18 semanas; 2). "Pirâmide", no qual inicia com pequenas doses, aumentando progressivamente até o ápice e, após atingir a dosagem 
máxima, existe a redução regressiva até o final do período; e 3). "Stacking", ou uso alternado de acordo com a toxicidade, referindo-se à utilização de vários esteróides ao mesmo tempo (MEIRELLES; MENEZES; WEFFORT, 2011).

Homens e mulheres utilizam EAA para o ganho de massa muscular, perda de peso ou melhoramento da aparência ou desempenho esportivo. Denominam-se diversas vezes tais substâncias de agentes ergogênicos ou recursos ergogênicos, no entanto, poderiam ser chamadas "bodyimagedrugs", uma vez que tais substâncias estão sendo mais utilizadas para melhoria da aparência do que para a melhora do desempenho em um esporte específico, mesmo tendo conhecimento que a melhora do desempenho não é justificativa para o uso de substâncias ilegais (ALMUKHTAR et al., 2015; PENDERGRAFT et al., 2014).

Segundo Silva et al. (2016), no Brasil é necessária a indicação médica para o uso de EAA, porém, em alguns casos essas drogas são vendidas em drogarias e farmácia de manipulação livremente. Atualmente a Portaria n ${ }^{\circ} 344$, de 12 de maio de 1998 disserta que o controle e fiscalização de produtos e comércio, manipulação e uso de EAA deverá ser realizada em conjunto com as autoridades do Ministério da Saúde (MS), Ministério da Fazenda e seus congêneres nos Estados, Municípios e Distrito Federal. No mais, a Lei ${ }^{\circ} 10.409 / 02$ de 11 de janeiro de 2002 relata sobre a prevenção, o tratamento, o controle e a repressão ao uso ilícito de substâncias que causem dependência química e psíquica. No ano de 2000 tornouse restrito a venda de esteroides anabolizantes através a lei promulgada pelo então Presidente Fernando Henrique Cardoso de $\mathrm{n}^{\circ} 9.965$ de 27 de abril, sendo permitida a venda destes EAA somente com apresentação e retenção de receita médica.

Segundo Ferreira et al. (2014), o uso indiscriminado de EAA é um problema sério de saúde pública em nível mundial, no qual o manejo de forma incorreta destes compostos pode, posteriormente, desenvolver efeitos colaterais, associados geralmente ao uso ilícito de megadoses, tais como hepatoxicidade, alterações no perfil lipídico no soro, retenção de líquido, hipertrofia cardíaca, hipogonodismo, ginecomastia em homens, hirsutismo e irregularidades menstruais. Deste modo, esses efeitos podem causar danos irreversíveis graves aos órgãos, além de ocasionar efeitos secundários, como aumento de pelos faciais, engrossamento da voz, aumento do clitóris, depressão, agressividade, queda de cabelo e psicose. Santos (2012) também considera um sério problema de saúde pública devido ao aumento significativo de pessoas que fazem o uso desses compostos e que podem desenvolver diversas comorbidades causadas por essas drogas, além dos usuários estarem mais suscetíveis ao envolvimento com outras drogas ilícitas, álcool e tabaco.

Quanto aos efeitos renais, para Venâncio et al. (2010), as megadoses de EAA ao longo e por algum período desencadeiam aumento nos índices de ureia e creatinina, lesionando os rins e, posteriormente, ocasionam a insuficiência renal. Logo, também preocupante do ponto de vista da saúde pública, pois ao afetar o sistema renal e causar a doença também acaba por onerar os cofres públicos, já que a doença, em fase $\mathrm{V}$ instalada requer Terapia Renal Substitutiva (TRS) podendo ser necessária hemodiálise, dialise peritoneal ou transplante renal quando recomendado (SILVA et al., 2016).

A respeito da Lesão Renal Aguda, segundo Hall (2017), esta apresenta-se da seguinte forma:

Anormalidades originadas no rim e que abruptamente diminuem o débito urinário se encaixam na categoria geral de injúria renal intrarrenal aguda. Essa categoria de injúria renal aguda pode ser dividida em (1) condições que afetam os capilares glomerulares ou outros vasos renais menores; (2) condições que lesam o epitélio tubular renal; e (3) condições que ocasionam lesão do interstício renal. Esse tipo de classificação se refere ao local primário da lesão, mas na medida em que a vasculatura renal e o sistema tubular em termos funcionais são interdependentes, o dano dos vasos sanguíneos renais pode levar a dano tubular, e o dano tubular primário pode levar a dano dos vasos sanguíneos renais (HALL, 2017, p. 258).

O uso de esteroides anabolizantes também pode desenvolver uma doença secundária denominada como glomerulonefrite, que se trata de um processo inflamatório nos rins, provocando perda de proteína e sangue através da urina e a partir disto, pode-se evoluir para o quadro de insuficiência renal crónica (PARENTE FILHO et al., 2020).

A glomerulonefrite não é uma única doença, mas um grupo de inflações que atingem o glomérulo, a parte dos rins responsável pela filtragem. Quando o glomérulo deixa de funcionar corretamente, o rim passa a produzir menos urina e as toxinas acabam ficando acumuladas no sangue. As glomeropatias podem ser divididas em quatro tipos: síndrome nefrítica aguda, glomerulonefrite rapidamente progressiva, síndrome nefrótica e glomerulonefriteoligossintomática (PARENTE FILHO et al., 2020). 
Ainda segundo o autor, quando a glomerulonefrite aparece, a pessoa fica edemaciada, principalmente na face e nas pálpebras, tende a reter a urina, manifestar hipertensão arterial, cefaleia, hipertermia, fadiga, náuseas, vômitos, dor abdominal e nas articulações, assim como apresentar perda de massa muscular (PARENTE FILHO et al., 2020).

Os danos causados pelo o uso de anabolizantes esteroides utilizados de forma indiscriminada e sem acompanhamento adequado, além de proporcionar um aumento na prevalência e na incidência de DRC em jovens e adultos, também podem acarretar a necessidade de um transplante renal, assim como assumir o risco de desenvolver tipos de câncer. Robinson (2015), cita que os cânceres associados ao uso abusivo de EAA incluem órgãos como o fígado, testículo, próstata, além de poder surgir à leucemia.

Estudos realizados apontam que o uso de esteroides anabolizantes também desenvolve o surgimento da Insuficiência Renal Aguda (IRA) em pacientes acometidos de doenças hepáticas que frequentemente ocorre devido à diminuição da perfusão renal por depleção intravascular, necrose, injuria tubular e síndrome hepatorenal. Anteriormente, a IRA nesses pacientes com doenças hepáticas por vezes chegou a ser atribuída a entidades conhecida como nefrose colêmica ou nefropatia, e icterícia severa obstrutivas com níveis de bilirrubina anormais. Foi associada à IRA por lesão direta das células tubulares renais pelos ácidos biliares e pela obstrução dos nefros pelos cilindros biliares (PARENTE FILHO et al., 2020). Recentemente, mesmo este conceito de lesão por ácidos biliares não ter sido aceito pela comunidade nefrológica, tem sido atualmente renovado interesse na IRA no cenário de doença hepática. Os dados ainda relatam que pacientes submetidos à terapia de doenças terminais hepáticas com anabolizante Nandrolona, Testosterona, Metandrostenolona e suplementos como os shakes de proteína, tiveram os níveis de ureia e creatinina no soro aumentado, aumento na taxa de filtração glomerular (FG), alteração de $\mathrm{pH}$ urinário, albumina e cristais. $\mathrm{O}$ mecanismo de ação de lesão renal por hiperbilirrubinemia severa é muito mais amplo que apenas um conceito hemodinâmico, porque a perfusão renal é diminuída semelhante à síndrome hepatorena e a fisiologia cirrótica contribui diretamente para a lesão renal, mas uma alta carga de ácidos biliares também induz a lesão tubular, pois esses ácidos são reabsorvidos nas células tubulares proximais que prejudicam a estrutura e a função destas células, essas deformidades podem ser observadas através de microscopia de urina e em biopsia de células renais (RANDY et al., 2014).

\section{CONCLUSÃO}

Estudos sobre a perda da função renal em jovens e adultos usuários de esteroides anabolizantes sem acompanhamento especializado são escassos, especialmente na literatura científica nacional. Contudo, os poucos dados encontrados reportam que o uso indiscriminado EAA podem causar lesões diversas ao organismo humano quando não acompanhados por um especialista. Quanto ao aparelho renal, este pode sofrer maiores danos, uma vez que é responsável pela filtragem e eliminação de impurezas do organismo.

O uso terapêutico dos EAA deve ser utilizado por indivíduos que requerem seu uso e serem acompanhados por profissionais capacitados para sua prescrição, pois seu uso indiscriminado já é considerado um problema de saúde pública. $\mathrm{Na}$ atualidade, com o crescente uso dos EAA, é necessário que haja maior inserção da educação em saúde nos diversos meios de obtenção de informações de qualidade, priorizado uma educação preventiva. 


\section{REFERÊNCIAS}

ALMUKHTAR, S. E.; ABBAS, A. A.; MUHEALDEEN, D. N.; HUGHSON, M. D. Acute kidney injury associated with androgenic steroids and nutritional supplements in bodybuilders $(\dagger)$. Clin Kidney J., v. 8, n. 4, p. 415-419, ago. 2015. Disponível em: <https://www.ncbi.nlm.nih.gov/pmc/articles/PMC4515889/ $>$. Acesso em 22 fev. 2021.

DOI: $10.1093 / \mathrm{ckj} / \mathrm{sfv} 032$.

BASTOS, M. G.; BREGMAN, R.; KIRSZTAJN, G. M. Doença renal crônica: frequente e grave, mas também prevenível e tratável. Rev. Assoc. Med., São Paulo, v. 56, n. 5, p. 248-253, 2010. Disponível em: <http://www.scielo.br/scielo.php?script=sci_arttext\&pid=S 0104-42302010000200028\&lng=en\&nrm=iso>. Acesso em: 10 out. 2018. https://doi.org/10.1590/S010442302010000200028 .

DAVANI-DAVARI, D.; KARIMZADEH, I.; KHALILI, H. The potential effects of anabolic-androgenic steroids and growth hormone as commonly used sport supplements on the kidney: a systematic review. BMC Nephrol., v. 20, n. 1, p. 1-12, mai. 2019. Disponível em: <https://bmcnephrol.biomedcentral.com/articles/10.1186/s1 2882-019-1384-0>. Acesso em: 22 fev. 2021. DOI: https://doi.org/10.1186/s12882-019-1384-0.

FELÍCIO, L. Os esteroides androgênicos anabolizantes e a educação física. São Paulo: Editora Schoba, 2010. 84 p.

FERREIRA, N. A. F.; SÁ, S. G. V.; ALEIXO, I. B.; PAIVA, A. M. R. Estudos dos efeitos provocados pelo uso do anabolizante Estanozolol. Brazilian of Journal of Surgery and Clinical Research, Belo Horizonte, v. 7, n. 3, p. 17-23, ago. 2014. Disponível em: <https://www.mastereditora.com.br/periodico/20140731_23 5533.pdf>. Acesso em: $21 \mathrm{fev} .2021$.

GRACELI, J. B. et al. Uso crônico de decanoato de nandrolona como fator de risco para hipertensão arterial pulmonar em ratos Wistar. Rev. Bras. Med. Esporte, Niterói, v. 16, n. 1, p. 46-50, fev. 2010. Disponível em: http://www.scielo.br/scielo.php?script=sci_arttext\&pid=S15 17-86922010000100009\&lng=en\&nrm=iso. Acesso em: 10 out. $2018 . \quad$ https://doi.org/10.1590/S151786922010000100009 .

HALl, J. E.; GUYTON, A. C. Tratado de Fisiología Médica. 13. ed. Rio de Janeiro: Elsevier, 2017. 1176 p.
KUMAR, V.; ABBAS, A. K.; ASTER, J. C. Robbins Patologia Básica. 9. ed. Rio de Janeiro: Elsevier, 2013. 928 p.

MEIRELLES, M.; MENEZES, S. P.; WEFFORT, V. R. S. O uso de agentes ergogênicos na adolescencia. Revista Médica de Minas Gerais, Minas Gerais, v. 21, n. 3 Supl. 1, p. 121144, 2011. Disponível em: <file:///C:/Users/User/Downloads/v21n3s1a30.pdf>. Acesso em: 10 out. 2018.

NEVES, P. D. M. M.; SESSO, R. C. C.; THOMÉ, F. S.; LUGON, J. R.; NASCIMENTO, M. M. Brazilian dialysis survey 2019. Braz. J. Nephrol., São Paulo, 2021. Disponível em: <http://www.scielo.br/scielo.php?script=sci_arttext\&pid=S 0101-28002021005010303\&lng=en\&nrm=iso > . Acesso em: 22 fev. 2021. http://dx.doi.org/10.1590/2175-8239-jbn2020-0161.

NEVES, P. D. M. M.; SESSO, R. C. C.; THOMÉ, F. S.; LUGON, J. R.; NASCIMENTO, M. M. Censo Brasileiro de Diálise: análise de dados da década 2009-2018. Braz. J. Nephrol., São Paulo, v. 42, n. 2, p. 191-200, jun. 2020. Disponível em: <http://www.scielo.br/scielo.php?script=sci_arttext\&pid=S 0101-28002020000200191\&lng=en\&nrm=iso > . Acesso em: 22 fev. 2021. https://doi.org/10.1590/2175-8239-jbn-20190234 .

PARENTE FILHO, S. L. A. et al. Kidney disease associated with androgenic-anabolic steroids and vitamin supplements abuse: Be aware! Nefrologia, v. 40, n. 1, p. 26-31, jan. - fev. 2020. Disponível em: <https://www.revistanefrologia.com/es-kidney-diseaseassociated-with-androgenicanabolic-articuloS0211699519301419>. Acesso em: 22 fev. 2021. DOI: 10.1016/j.nefro.2019.06.003.

PENDERGRAFT, W. F. III; HERLITZ, L. C.; THORNLEY-BROWN, D.; ROSNER, M.; NILES, J. L. Nephrotoxic effects of common and emerging drugs of abuse. Clin. J. Am. Soc. Nephrol., v. 9, n. 11, p. 1996-2005, nov. 2014. Disponível em: <https://www.ncbi.nlm.nih.gov/pmc/articles/PMC4220747/ >. Acesso em: 22 fev. 2021. DOI: 10.2215/CJN.00360114.

RANDY, L.; CASTANO E., MOECKEL, G.; PERAZELLA, M. A. Nefropatias por ácidos biliares em um fisiculturista por abuso de esteroides anabolizantes 
androgénicos. American Journal of Kidney Diseases, Estados Unidos, v. 64, n. 3, p. 473-476, 2014.

RIELLA, M. C. Princípios de Nefrologia e Distúrbios Hidreletrolíticos. 6. ed. Rio de Janeiro: Guanabara Koogan, 2018. $1136 \mathrm{p}$.

ROBINSON, T. R. Genética para leigos. 2. ed. Rio de Janeiro: Alta Books Editora, 2015. 352 p.

SANTOS, L. F. O uso de esteroides androgênicos anabolizantes nas academias de musculação da zona sul de Porto Velho. 2012. 48 f. Monografia de Graduação Curso de Educação Física, Universidade Federal de Rondônia, Porto Velho, 2012. Disponível em: <https://livrozilla.com/doc/609278/o-uso-de-esteroidesandrog\%C3\%AAnicos-anabolizantes-nas-academ>. Acesso em: 20 jan. 2019.
SILVA, S. B.; CAULLIRAUX, H. M.; ARAUJO, C. A. S.; ROCHA, E. Uma comparação dos custos dos transplantes renal em relação às dialises no Brasil. Cad. Saúde Pública, Rio de Janeiro, v. 32, n. 6, e00013515, 2016. Disponível em: $<$ http://www.scielo.br/scielo.php?script=sci_arttext\&pid=S 0102-311X2016000605005\&lng=en\&nrm=iso>. Acesso em: 20 jan. 2019.

VENÂNCIO, D. P.; NOBREGA, A. C. L.; TUFIK, S.; MELLO, M. T. Avaliação descritiva sobre o uso de esteroides anabolizantes e seu efeito sobre as variáveis bioquímicas e neuroendócrinas em indivíduos que praticam exercício resistido. Rev. Bras. Med. Esporte, Niterói, v. 16, n. 3, p. 191-195, jun. 2010. Disponível em: <http://www.scielo.br/scielo.php?script=sci_arttext\&pid=S 1517-86922010000300007\&lng=en\&nrm=iso >. Acesso em: 10 out. 2018. https://doi.org/10.1590/S151786922010000300007. 